\title{
Investigating oxidative stability of lithium-ion battery electrolytes using synthetic charge-discharge profile voltammetry
}

\author{
Alma Mathew ${ }^{1}$, Matthew J Lacey ${ }^{2}$, Daniel Brandell ${ }^{1 *}$ \\ ${ }^{1}$ Department of Chemistry - Ångström Laboratory, Uppsala University, Box 538, SE-75121 \\ Uppsala, Sweden \\ ${ }^{2}$ Scania CV AB, SE-15187 Södertälje, Sweden \\ *Corresponding author. E-mail: daniel.brandell@kemi.uu.se
}

\begin{abstract}
Electrolytes are an integral part of any electrochemical energy storage systems, including batteries. Among the many properties which determine the applicability of a Li-ion battery electrolyte, electrochemical stability - and for high voltage electrodes, in particular anodic stability - is a key parameter to consider. Despite being simple and straightforward to employ, the conventional linear sweep voltammetry (LSV) technique often leads to an over-estimation of the oxidative stability. In this study, an alternative approach termed Synthetic Charge-discharge Profile Voltammetry (SCPV) is explored to investigate the oxidative electrolyte stability. We have found this to be a convenient method of quantifying the anodic stability of the electrolyte in a more practically representative manner, in which passivation kinetics and electrode potential changes at the electrode-electrolyte interface are more appropriately reproduced. The viability of this technique is explored with liquid electrolytes based on ether, carbonate, sulfone and carbonate-sulfone mixtures, all with lithium hexafluorophosphate $\left(\mathrm{LiPF}_{6}\right)$ salt, tested for a potential profile equivalent to $\mathrm{LiNi}_{0.5} \mathrm{Mn}_{1.5} \mathrm{O}_{4}$ electrodes. The credibility of this technique is validated by correlations to the coulombic efficiencies of corresponding half-cells.

Keywords: Li-ion battery, electrolyte, electrochemical stability, voltammetry, $\mathrm{LiNi}_{0.5} \mathrm{Mn}_{1.5} \mathrm{O}_{4}$.
\end{abstract}

\section{Introduction}

Battery energy density is a decisive factor for electrification of technologies such as electric vehicles, and often constitutes a key parameter when comparing different battery chemistries. A current strategy within lithium-ion battery research is identifying cathode materials with higher operating potential, while preserving capacity and maintaining safety.[1,2] An additional advantage of a high-voltage cell 
is a lower current required to deliver the same power, which can reduce resistive $\left(I^{2} R\right)$ losses; similarly, on the system level, a smaller number of cells is required to reach the pack voltage, which can further reduce losses and complexity in the pack. A critical challenge is, however, that such high voltage cathodes need electrolyte systems that are electrochemically stable to prevent electrolyte oxidation throughout cell operation; i.e., an electrochemical stability window (ESW) which is adopted for the electrode materials of choice.[3] Alternatively, the electrolyte decomposition products must effectively passivate the positive electrode, similar to the Solid Electrolyte Interphase (SEI) formed on the anode, thereby rendering a kinetically stable cycling system.[4] However, this process is well-understood to lead to a consumption of electrolyte and build-up of internal resistance, and therefore an intrinsically stable electrolyte is preferred.[5] Determining the ESW of the battery electrolyte, and particularly the oxidative stability when applying a high voltage cathode, is thus important to prevent unwanted electrolyte decomposition. Recent research - both computational and experimental - has in this context highlighted difficulties and shortcomings in several approaches for estimating the ESW.[6-8]

For example, linear sweep voltammetry (LSV) is a well-established technique to determine the electrochemical stability of electrolytes, and is extensively used by the battery research community. Generally applied in a two-electrode setup, the potential is swept linearly across the working electrode and the counter electrode while measuring the current produced at the working electrode with time.[9] The stability limit is determined as the onset of a larger current response, although such an onset is often arbitrarily defined and there is no theoretical background for how to distinguish it. For example, a wide variety of different cut-off current densities have been chosen in literature, ranging from $10 \mu \mathrm{A} / \mathrm{cm}^{2}$ to the most typical $1.0 \mathrm{~mA} / \mathrm{cm}^{2}$. In addition to obtaining the oxidative stability limit using a defined onset current density, another popular way of interpreting the LSV curve is to use an onset potential at the intersection point of the tangent drawn at the maximum slope of current density.[10] The oxidative stability limit has also been identified in the literature as the potential intercept of the initial rise, rather than the exponential increase in current density.[11] However, depending on the shape of the curve and the strength of the current response, such a procedure may often not be acceptable. Moreover, parameters such as the voltage scan rate, nature and inertness of the working electrode, and its surface area, might differ substantially from the conditions under which the actual battery work, which will influence the electrochemical response. In scientific literature, a wide span of LSV scan rates ranging from $0.01 \mathrm{mV} / \mathrm{s}$ to $10 \mathrm{mV} / \mathrm{s}$ are frequently found, but are often uncorrelated to any practical battery operation.[12, 13]

Platinum, stainless steel, several forms of carbon-based electrodes such as glassy carbon, graphite, etc., are generally used as the working electrodes in LSV experiments.[14-16] However, using the conventional non-porous $\mathrm{Pt}$ or glassy carbon electrodes generates, as pointed out by $\mathrm{Xu}$ et al., ${ }^{[3]}$ electrolyte stability data with a poor correspondence to the anodic stability of an electrolyte with a real working electrode like $\mathrm{Li}_{x} \mathrm{Mn}_{2} \mathrm{O}_{4}$. While the electrochemical stability of a specific electrolyte is, at least in theory, a thermodynamic quantity, several experimental conditions play a vital role in practical LSV 
measurements which causes discrepancies when comparing 'inert' electrodes with porous electrode systems.[8] For example, catalytic effects by the electrode material employed in a real battery are not taken into account in conventional LSV measurement set-ups. Differences in surface properties such as area and porosity of the working electrode are here also important factors, which are normally not being addressed. These factors determine the number of reaction sites and consequently affect the reaction kinetics as more sites allow increased reactivity in a given amount of time.[17] There also exist other crucial differences between LSV experiments and the electrochemical conditions in true Li-ion cells, which will influence the practical stability. For example, it has been observed that the aluminium current collector used at the positive electrode is prone to corrosion even with $\mathrm{LiPF}_{6}$ salt in an $\mathrm{LiFePO}_{4}$ system, [18] despite operating at a relatively low voltage plateau of $3.5 \mathrm{~V}$ vs $\mathrm{Li} / \mathrm{Li}^{+}$. Moreover, the ratio of volume of electrolyte to the active material in a cell can have considerable contribution to the ratio of impurities to both the active surface and decomposition products, and also varies considerably between LSV experiment set-ups and Li-ion cells.[19] Altogether, neglecting these aspects in the LSV measurements generally overestimates the ESW in the Li-ion cell environment. The interpretation of LSV experiments, considering the above-described factors, is therefore often arbitrary. This factor, combined with the basic differences in cell construction and experimental conditions compared to the intended application, means that the ESW is typically overestimated, leading to a misleading picture of interfacial stability and poor correlation with battery-specific measures such as coulombic efficiency and capacity retention.[3, 20, 21]

In this work, we explore an alternative approach in terms of a Synthetic Charge-discharge Profile Voltammetry (SCPV) method as a technique for determining the oxidation stability of four different electrolytes - tetraethylene glycol dimethyl ether (TEGDME), ethylene carbonate: diethyl carbonate (EC: DEC), sulfolane and a mixture of EC: DEC and sulfolane $(1: 1 \mathrm{v} / \mathrm{v})$, all with $\mathrm{LiPF}_{6}$ salt - when targeting their use with the high-voltage $\mathrm{LiNi}_{0.5} \mathrm{Mn}_{1.5} \mathrm{O}_{4}$ (LNMO) Li-ion battery cathode material. In contrast to an LSV measurement, the SCPV method controls the potential so that it follows a specific potential profile of interest (here of the LNMO active material), thereby recreating realistic operational conditions at least in terms of potential changes with time, which will influence the kinetics of the reactions which may take place and any subsequent passivation processes. This makes the experiment more directly comparable with the corresponding half-cell data, and should thereby lead to a more realistic determination of the ESW.

\section{Experimental}

Polycrystalline disordered type LNMO cathode material (TBM-129) with spherical particles (average diameter 5-20 $\mu \mathrm{m}$ ) was received from Haldor Topsoe A/S and used as received. LNMO electrodes were made with the composition of $85 \mathrm{wt} \%$ LNMO, 5 wt.\% carbon black (C-ENERGY Super C65, Imerys) and 10 wt.\% poly(vinylidene difluoride-hexafluoropropylene) (PVdF-HFP, Kynar FLEX 2801, 
Arkema) dissolved in N-methyl pyrrolidone (NMP, Sigma Aldrich). The slurry was homogenized using a shaker ball mill for $30 \mathrm{~min}$ and was cast on a carbon-coated aluminium foil (20 $\mu \mathrm{m}$ thick, MTI). The electrodes were dried at $70{ }^{\circ} \mathrm{C}$ for $12 \mathrm{~h}$ and thereafter cut into $13 \mathrm{~mm}$ diameter discs, and were dried additionally in vacuum at $120{ }^{\circ} \mathrm{C}$ in a Büchi oven in an argon-filled glovebox $\left(\mathrm{O}_{2}<1 \mathrm{ppm}, \mathrm{H}_{2} \mathrm{O}<1\right.$ ppm). Four types of electrolytes were used in the study. $1 \mathrm{M} \mathrm{LiPF}_{6}$ EC: DEC (1:1 w/w) (LP40, Gotion) electrolyte was used as received. For the others, $\mathrm{LiPF}_{6}$ (Sigma Aldrich, > 99.99\%) salt was dried at 120 ${ }^{\circ} \mathrm{C}$ for $12 \mathrm{~h}$ under vacuum to prepare electrolytes with sulfolane (Sigma Aldrich, 99\%) and TEGDME (Sigma Aldrich, 99\%) solvents. A fourth electrolyte was made by mixing LP40 and $1 \mathrm{M} \mathrm{LiPF}_{6}$ sulfolane in a 1:1 volume ratio. Preparation of $1 \mathrm{M} \mathrm{LiPF}_{6}$ in sulfolane, $1 \mathrm{M} \mathrm{LiPF}_{6}$ sulfolane:LP40 (1:1 v/v) mixture and $1 \mathrm{M} \mathrm{LiPF}_{6}$ in TEGDME electrolytes took place inside the glovebox. Monolayer microporous polypropylene separators (Celgard 2500, $16 \mathrm{~mm}$ diameter) and Whatman glass fibre separators (16mm diameter) were also dried under vacuum at $70{ }^{\circ} \mathrm{C}$ for $5 \mathrm{~h}$. Two-electrode half-cells were assembled in coin cell format (CR2025, Ni-plated) with metallic lithium (15 mm diameter, $125 \mu \mathrm{m}$ thick, Cyprus Foote Mineral) as counter/reference electrode. Two Celgard separators soaked with $80 \mu \mathrm{L}$ electrolyte were used in between the working and counter electrode, in all cells except the $1 \mathrm{M} \mathrm{LiPF}_{6}$ sulfolane which used glass fibre.

The LNMO|LP40|Li cells were cycled at a C/10 rate at room temperature between 3.5 and $5 \mathrm{~V}$ to obtain the voltage profile using an Arbin BT cycler. The obtained LNMO charge profile was then imported to the voltammetric measurements employing the SCPV method. All SCPV method experiments were carried out using a VMP2 potentiostat (BioLogic) at room temperature.

\section{Results and discussion}

The oxidative stability of all the four electrolytes used in this study, $1 \mathrm{M} \mathrm{LiPF}_{6}$ TEGDME, $1 \mathrm{M} \mathrm{LiPF} 6$ sulfolane, LP40 and LP40:1M LiPF 6 sulfolane $(1: 1 \mathrm{v} / \mathrm{v})$ mixture, were measured using the traditional LSV technique with a voltage scan rate of $0.1 \mathrm{mV} / \mathrm{s}$ vs. Li. The motivation behind choosing these particular electrolytes was that they together span common lithium-ion battery electrolyte solvent functionalities. Furthermore, LP40 is a carbonate-based electrolyte that is the most commonly used for the Li-ion systems,[22-24] sulfolane: $\mathrm{LiPF}_{6}$ is often claimed to be more oxidatively stable,[25] while ether-based systems such as TEGDME:LiPF 6 are is often described as oxidatively less stable and is therefore expected to display more rapid decomposition.[26] The mixture of LP40 and sulfolane:LiPF 6 is employed primarily as a bench-marking system, and is expected to have intermediate properties of the two components. The LSV measurements were performed in a two-electrode coin cell format with carbon-coated aluminium foil as the working electrode and lithium metal as the counter electrode. From the LSV curve of TEGDME:LiPF 6 shown in Figure 1, it is observed that the current density constantly increases up to $4.7 \mathrm{~V}$ and then increases rapidly until the upper cut-off voltage is reached. This indicates that the oxidation of TEGDME:LiPF 6 is rapid above $\sim 4.7 \mathrm{~V}$; however, this also highlights that the exact 
voltage point of the beginning of the decomposition reaction is difficult to identify from this curve. If considering the voltage point just before the exponential rise in current density would, it would lead us to presume that the electrolyte is stable up to $\sim 4.7 \mathrm{~V}$ vs. $\mathrm{Li}$, but this conclusion can certainly be questioned considering the current density observed at lower voltages.

For the case of the other electrolytes, i.e. sulfolane:LiPF 6 , LP40 and mixed LP40:sulfolane, there is a negligible rise in current density up to $\sim 4.1 \mathrm{~V}$. But, beginning from $\sim 4.2 \mathrm{~V}$, a rise in current can be observed in the case of sulfolane: $\mathrm{LiPF}_{6}$, which stabilizes from $4.25 \mathrm{~V}$ until $4.33 \mathrm{~V}$. Another peak can be seen in the range 4.33-4.54 V, which thereafter it stabilizes up to $4.78 \mathrm{~V}$, after which the current density increases exponentially. Similarly, a peak appears in the range 4.13-4.3 V for the LP40 electrolyte. Then a smaller current increase appears until it starts to go up rapidly from $\sim 4.51 \mathrm{~V}$, after which it further stabilizes again until $\sim 4.71 \mathrm{~V}$. It is therefore also hard to conclude the oxidative stability of LP40 in this regard. The LSV curve of the mixture, LP40:sulfolane, follows as expected somewhat of an intermediate trend between that of LP40 and sulfolane:LiPF . The voltammogram indicates that one could expect the electrolyte mixture to have higher oxidative stability than $\mathrm{LP} 40$ and sulfolane:LiPF 6 until $4.75 \mathrm{~V}$, from where the current density crosses the value of the sulfolane: $\mathrm{LiPF}_{6}$ electrolyte and increases considerably.

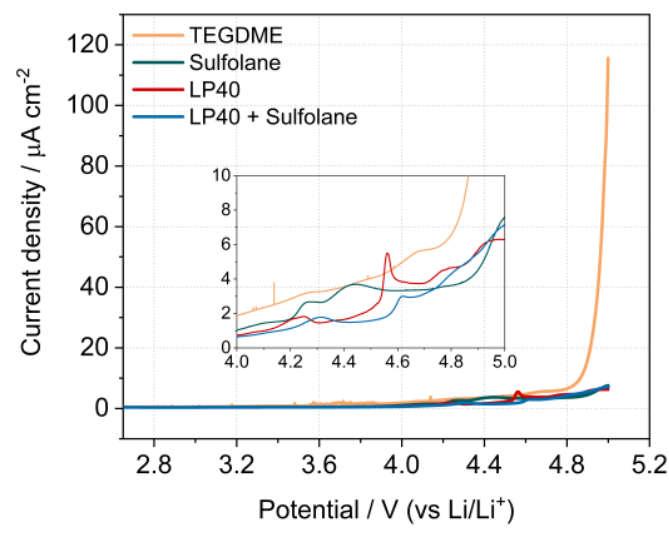

Figure 1. Linear sweep voltammograms of $\mathrm{Li}$ | electrolyte | C-coated Al foil cells from OCV up to $5 \mathrm{~V}$ vs. Li using a scan rate of $0.1 \mathrm{mV} / \mathrm{s}$. The inset shows a magnification of the data obtained between 4 and $5 \mathrm{~V}$ vs. Li.

To explore the oxidative stability of these electrolytes further, the high voltage cathode system of LNMO was chosen. The charge and discharge voltage profiles of the LNMO half-cell shown in Figure 2 was obtained with LP40 electrolyte cycled at room temperature at a C/10 rate. This charge-discharge voltage profile of LNMO was then used in the SCPV method to explore its applicability, in comparison to LSV. The potential was controlled to follow the electrode potential profile of LNMO. 


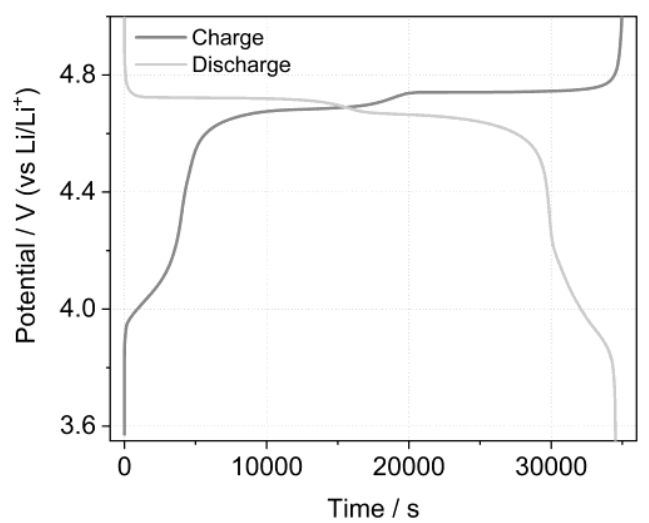

Figure 2. Charge and discharge voltage curves of the $5^{\text {th }}$ cycle of an LNMO half-cell.

The charge vs. potential (Q vs. E) plot and the differential capacity (dQ/dE vs. E) plot using this SCPV method for all four electrolyte systems is shown in Figure 3. It should be noted that it is the accumulated charge passed, rather than the current, which is displayed on the y-axis (the voltammograms of the first anodic sweep of the four electrolytes is shown in Figure S2). By plotting the data this way, it is easy to identify and pinpoint the Faradaic reactions occurring during the potential sweep: capacitive processes account for a relatively small portion of the passed charge, in the differential capacity plot only noncapacitive processes are represented as peaks (for purely capacitive processes, dQ/dE is a constant). For each Q vs. E curve, a step-wise increase in the charge passed are observed for all electrolytes, first at 4.0-4.1 $\mathrm{V}$ and then more significantly at $\sim 4.7 \mathrm{~V}$. The potentials at which these charge step increases occur corresponds to the potential plateaus of the LNMO potential profile itself (Figure 2), indicating that the total charge passed during each sweep is not a continuous process but rather depends significantly on time spent at each voltage level, especially at higher potentials. In Figure 2, a minor plateau in the 4.0-4.1 V region, corresponding to the $\mathrm{Mn}^{3+} / \mathrm{Mn}^{4+}$ redox couple is observed, which is a feature of disordered LNMO.[27] Much more pronounced plateaus are observed at $\sim 4.67 \mathrm{~V}$ and $\sim 4.74$ $\mathrm{V}$ corresponding to the $\mathrm{Ni}^{2+} / \mathrm{Ni}^{3+}$ and $\mathrm{Ni}^{3+} / \mathrm{Ni}^{4+}$ redox couples, respectively. As can be seen in Figure 2 , the cell spends considerably more time at the voltage plateaux than in any other regions in the voltage profile. Thereby, the sharp increase in the amount of charge in the Q vs. E curves, e.g. at $\sim 4.67 \mathrm{~V}$ and at $\sim 4.74 \mathrm{~V}$ in Figure. 3a, indicates a higher amount of time spent at these higher voltages. This specific feature of spending an equal amount of time using this voltammetric technique as in a galvanostatic cycling experiment is an advantage of the SCPV method.

Interestingly, it can be observed from Figure $3 \mathrm{a}$ that the sulfolane:LiPF 6 system passes more charge in the potential range of 3.97-4.16 V compared to other electrolytes when it follows the potential profile of LNMO. This charge passed in this potential range is attributed to the relatively long amount of time spent in this potential range in the LNMO profile, however it is noteworthy that this increase is, unexpectedly, only significantly visible in the sulfolane: $\mathrm{LiPF}_{6}$ electrolyte, which is often understood to 
have higher oxidation stability than the reference carbonate electrolyte. This increase in charge stabilizes between $\sim 4.1$ and $\sim 4.4 \mathrm{~V}$, after which a sharp rise for the charge is observed until $4.74 \mathrm{~V}$. Surprisingly, the TEGDME:LiPF 6 electrolyte by comparison passes relatively less amount of charge in the lower plateau region $(<4.3 \mathrm{~V})$, compared to the sulfolane: $\mathrm{LiPF}_{6}$ electrolyte, but the charge increases significantly higher in the higher plateau region $(>4.3 \mathrm{~V})$. Furthermore, based on the LSV results in Figure 1, one would expect the LP40 electrolyte to pass a relatively higher amount of charge than the sulfolane-based counterpart, as the current density of LP40 also increases significantly with voltage starting from $4.4 \mathrm{~V}$ up to $4.96 \mathrm{~V}$. However, from the data obtained using the LNMO voltage profile and the SCPV technique, it can be observed that the amount of charge at the end of the first charge is more than double for sulfolane:LiPF $6: 15.68 \mu \mathrm{Ah} \mathrm{cm}^{-2}$ as compared to $7.02 \mu \mathrm{Ah} \mathrm{cm}^{-2}$. The LP40:sulfolane mixture shows an intermediate value between the sulfolane-based and the LP40 electrolytes of the total amount of charge passed during the first charge $\left(8.72 \mu \mathrm{Ah} \mathrm{cm}^{-2}\right)$, although this value and the overall Q vs. E curve more resembles that of LP40.
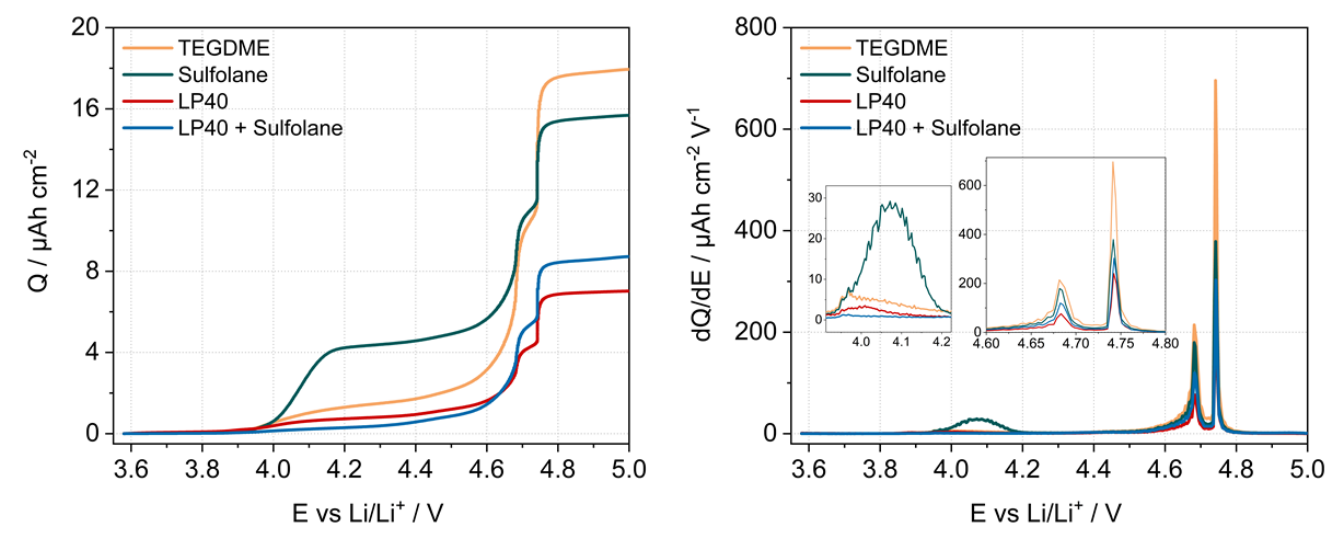

Figure 3. a) Amount of charge passed in the first charge obtained using the SCPV technique (Q vs. E plot), and b) change of $\mathrm{dQ} / \mathrm{dE}$ as function of $\mathrm{E}$. Insets show magnifications in the ranges 3.9-4.2 $\mathrm{V}$ and 4.5-4.8 V, respectively.

Figure $3 \mathrm{~b}$ presents the differential plot of Figure $3 \mathrm{a}$, to more easily identify the Faradaic processes occurring in the specific voltage range. Even though there is a gradual increase in the amount of charge passed in the case of TEGDME, it is not significantly larger than LP40 until $4.67 \mathrm{~V}$ where there is a sudden increase in charge passed. In Figure $3 \mathrm{~b}$, a peak at $4.67 \mathrm{~V}$ is observed for all electrolytes, where the integral corresponds to the total charge passed. Similarly, a peak at $4.74 \mathrm{~V}$ is observed for all electrolytes but with different intensities, which could be due to the higher amount of time spent at a potential corresponding to the $\mathrm{Ni}^{3+} / \mathrm{Ni}^{4+}$ redox plateau. Both of these peaks, at $4.67 \mathrm{~V}$ and $4.74 \mathrm{~V}$ indicate Faradaic reactions occurring due to the electrolyte decomposition. The difference in peak intensities could be attributed to the magnitude of degradation taking place, i.e. implying that a higher peak intensity corresponds to an electrolyte which is decomposing more severely. In this regard, TEGDME 
is showing the least oxidative stability and LP40 the best. The notable exception in Figure $3 b$ is the broad peak at the lower plateau region of that of sulfolane electrolyte compared to other electrolytes studied. This suggests that some amount of decomposition of sulfolane occurs at this particular voltage range, which compared to LP40 and TEGDME is higher. In addition, unlike sulfolane, which has a more symmetrical peak shape, LP40 and TEGDME exhibit asymmetrical peaks at the lower plateau, which rise early and fall more gradually, suggesting the presence of some passivation in the electrode.

With this technique of following the potential profile of the electrode material with controlled voltage, it can be noticed that the electrolyte decomposition is highly dependent on the voltage profile of the electrode, in contrast to the data appearing when applying a constant scan rate as in the case of LSV. Even though the SCPV technique could be used to identify several Faradaic reactions during the scan over large and relevant voltage ranges, it should also be noted that it is still difficult to pinpoint a particular voltage and specify it as an oxidative stability limit. The SCPV technique shows, however, that it allows relatively straightforward quantitative comparisons of different electrolytes through the evaluation of the total amount of charge passed. This technique can easily be employed also for other electrode voltage profiles, e.g. those corresponding to $\mathrm{LiFePO}_{4}$ (LFP), $\mathrm{LiNiMnCoO}_{2}$ (NMC), $\mathrm{LiNiCoAlO}_{2}$ (NCA), etc.

Considering the contradictory results obtained from LSV and SCPV techniques, galvanostatic cycling experiments were performed with LNMO electrodes using all four different electrolytes at a $\mathrm{C} / 10$ rate. The coulombic efficiency (CE) is often used as a predictor of the electrolyte stability during a galvanostatic cycling experiment, since it reflects the proportion of parasitic reactions taking place during cycling.[4] For this LNMO system, we expect that the coulombic efficiency largely reflects the extent of electrolyte oxidation, since loss of active material (LAM) has previously observed to be a relatively minor degradation mechanism.[28] As expected, the half-cell with TEGDME:LiPF 6 electrolyte performed the worst, with the cell failing in the very first charge (shown in Figure S3a). As can be seen, the TEGDME:LiPF 6 electrolyte fails to completely delithiate LNMO at both the lower plateau (4.0-4.1 V (vs Li/Li $\left.{ }^{+}\right)$) and upper plateau $\left(4.7 \mathrm{~V}\left(\mathrm{vs} \mathrm{Li} / \mathrm{Li}^{+}\right)\right)$, leading to a low specific capacity and subsequent cell failure. 


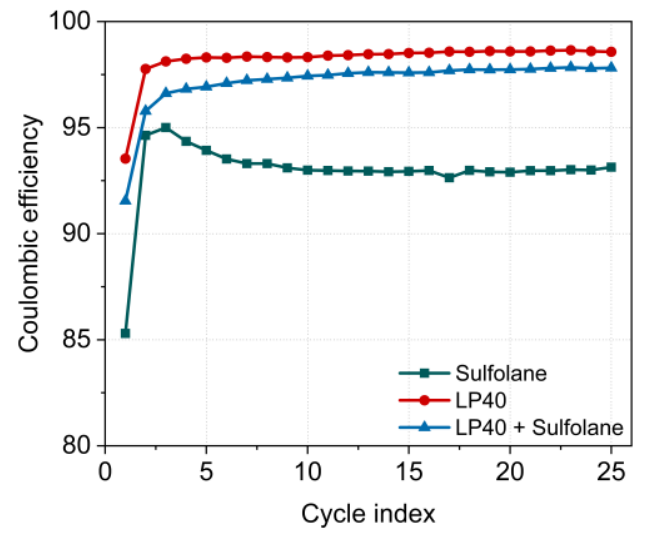

Figure 4. Coulombic efficiencies of LNMO half-cells.

The first cycle coulombic efficiency of the sulfolane-based electrolyte was $85.29 \%$, which is significantly lower than the CE (93.53\%) of the LP40-based cell or the intermediate first CE of the mixture (91.54\%). The CE during further cycles showed a similar trend, with LP40 displaying the best performance, followed by the LP40:sulfolane mixture and then sulfolane:LiPF 6 . As can be seen from the galvanostatic cycling results with LNMO half-cells, these results are in fair agreement with the SCPV results, provided that a higher amount of electrolyte oxidation can partly explain the lower CE.

\section{Using SCPV for multiple cycle analysis}

To further explore this voltammetry methodology, multiple SCPV cycles of charge and discharge using the LNMO voltage profile were performed with each of the four electrolytes to investigate any kind of passivation behaviour in the cells. The LNMO voltage profile was thereby divided into two regions: the lower plateau region $<4.3 \mathrm{~V}$ where the $\mathrm{Mn}^{3+} / \mathrm{Mn}^{4+}$ conversion occurs and the higher plateau region $>4.3$ $\mathrm{V}$ where the $\mathrm{Ni}^{2+} / \mathrm{Ni}^{4+}$ redox couple is active (Figure 5a). The amount of irreversible charge passed, i.e. the charge from the decomposition of the electrolyte, during each cycle in each respective region is presented in Figure 5 b-d (see Figure S5 of the Supporting Information for the amount of charge passed in the lower plateau region i.e., $<4.3 \mathrm{~V}$ ) for all of the four electrolytes used. 
a) $-\mathrm{Ch},<4.3 \mathrm{~V}-\mathrm{Ch},>4.3 \mathrm{~V}-\mathrm{Dch},<4.3 \mathrm{~V}-\mathrm{Dch},>4.3 \mathrm{~V}$

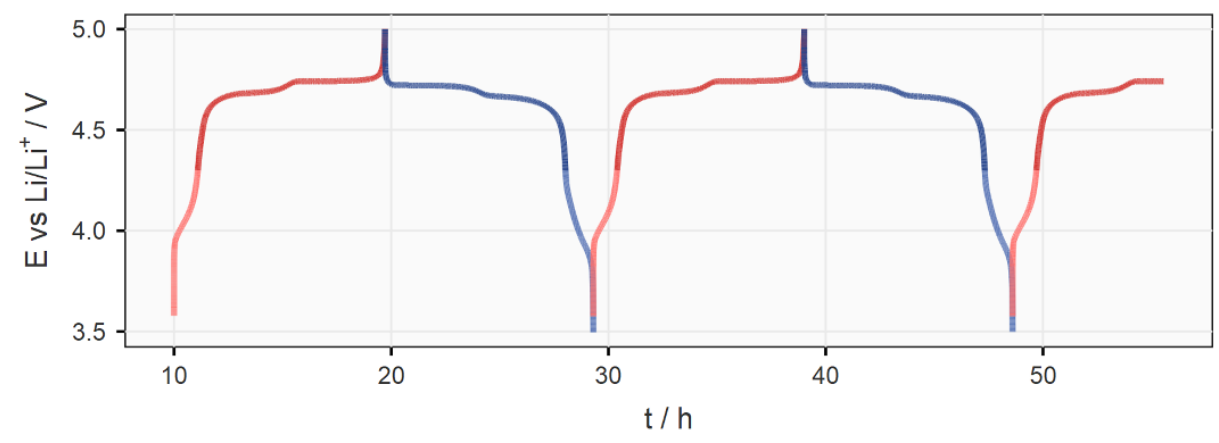

b) $\quad \square \mathrm{ch},<4.3 \vee \square \mathrm{ch}_{1}>4.3 \mathrm{~V} \square \mathrm{Dch},<4.3 \vee \square \mathrm{Dch},>4.3 \mathrm{~V}$

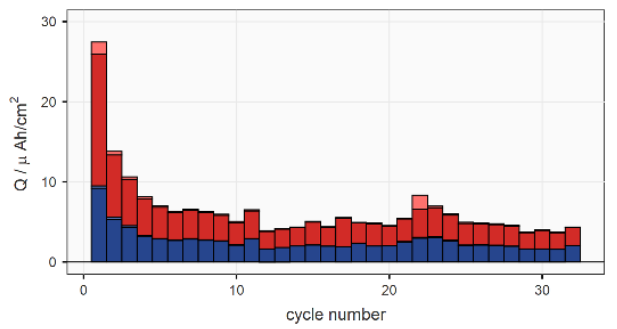

d) $\square \mathrm{Ch}_{1}<4.3 \vee \square \mathrm{Ch},>4.3 \vee \square \mathrm{Dch}_{1}<4.3 \vee \square \mathrm{Dch}_{1}>4.3 \mathrm{~V}$

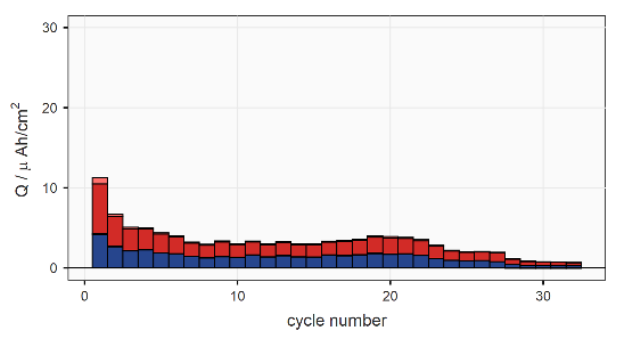

c) $\square \mathrm{Ch},<4.3 \vee \square \mathrm{Ch},>4.3 \vee \square \mathrm{Dch},<4.3 \vee \square \mathrm{Dch},>4.3 \mathrm{~V}$

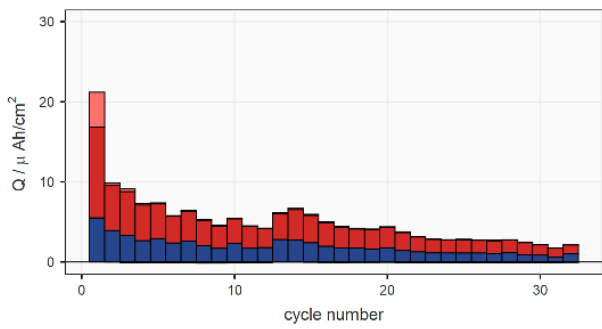

e) $\square \mathrm{ch},<4.3 \vee \square \mathrm{ch},>4.3 \vee \square \mathrm{Dch},<4.3 \vee \square \mathrm{Dch},>4.3 \mathrm{~V}$

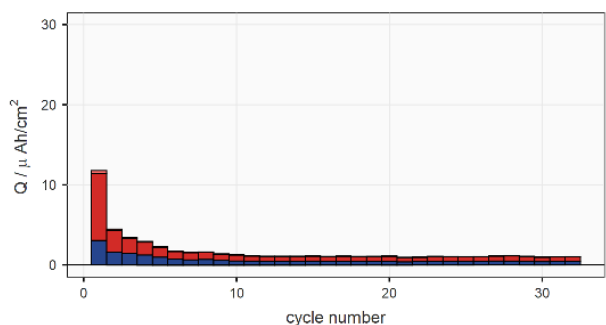

Figure 5. (a) LNMO half-cell voltage profile and multiple SCPV cycles of anodic and cathodic sweeps for (b) TEGDME:LiPF, (c) Sulfolane:LiPF, (d) LP40:LiPF 6 and (e) LP40:sulfolane mixture.

When analysing Figure 5 b-e, it can be observed that, as expected, TEGDME:LiPF 6 contributed to the highest amount of irreversible charge, $27.45 \mu \mathrm{Ah} \mathrm{cm}^{-2}$ in the first cycle, followed by sulfolane:LiPF 6 . This indicates that the irreversible oxidation in the first cycle is high in the case of TEGDME:LiPF 6 . It is also worth mentioning that the electrolyte oxidation continues also at the higher plateau region of the cathodic sweep, and it is significantly higher for the TEGDME:LiPF 6 than for other electrolytes. The total amount of irreversible charge in the second cycle drops to $13.8 \mu \mathrm{Ah} \mathrm{cm}^{-2}$; approximately half of that in the first cycle. It does not appear to stabilize even after 30 cycles, indicating ongoing oxidation of the electrolyte and comparatively weak passivation of the electrode. When comparing the sulfolane: $\mathrm{LiPF}_{6}$ electrolyte to other electrolyte systems, a clear distinction is the increased amount of charge passed in the lower plateau region. Similar to TEGDME:LiPF 6 , sulfolane:LiPF 6 displays a reduction in the total charge passed during the second cycle, while the amount of charge passed in subsequent cycles also implies similarly poor passivation behaviour. The cells with LP40 and the mixture of LP40 and sulfolane:LiPF 6 electrolytes, on the other hand, showed a total amount of charge at 
the end of the first cycle that was both similar to each other and lower than the other electrolytes; the mixture exhibiting only $0.47 \mu \mathrm{Ah} \mathrm{cm}^{-2}$ higher than LP40. In the first cycle cathodic sweep, the amount of charge produced during the oxidation of the LP40:sulfolane electrolyte mixture is actually lower than with LP40. Furthermore, starting from the second cycle, the LP40:sulfolane electrolyte mixture displays a decreased total amount of charge and appears to reach a steady charge value, implying the formation of a stable passivation layer.

The galvanostatic cell cycling and multiple SCPV cycling using the same electrolytes show some significant differences. For example, when the TEGDME:LiPF 6 electrolyte is used in an LNMO halfcell, the cell fails already during the first cycle, but when the carbon-coated aluminium foil is used as the working electrode in SCPV cycles, the cell continues to function. Similarly, in the SCPV cycle measurements, the LP40-sulfolane electrolyte mixture shows a better passivation than the LP40 electrolyte, despite the fact that the LP40 based LNMO half-cell seems to have a greater coulombic efficiency. These differences suggest that the properties of the working electrode, particularly the active material, plays a decisive role for determining the stability of an electrolyte. Generally, one of the major challenges using these voltammetric techniques is that the working electrode used does not mimic the LNMO electrode well, and is thus ignoring any effects from surface area and catalytic effects. Moreover, since voltammetric measurements of Li-ion battery electrolytes conventionally use metallic lithium as the counter electrode, any electrolyte decomposition effects arising from the lithium side cannot be separated from the rest of the data. The results of this study thus show that the electrochemical stability of the battery electrolyte is ultimately highly reliant on the system employed and experimental conditions, and that the electrolyte ESW is not a factor which can be defined for all battery systems in general.

\section{Conclusions}

We have here demonstrated the applicability of a SCPV technique to evaluate the electrochemical stability of Li-ion battery electrolytes, using the high-voltage LNMO system as an example. This method is based on subjecting a polarizable electrode to the specific potential profile of any active material of interest, allowing a prediction of the practical electrochemical stability in electrochemical cells. When correlated to the coulombic efficiencies obtained in the galvanostatic cycling of the corresponding cells, a more representative trend was obtained as compared to standard LSV. Moreover, when employing this method for multiple cycles, the amount of irreversible charge formed during each cycle could be determined, allowing for a better understanding on the passivation behaviour, with the caveat that the effects arising from any specific chemistry of the "real" electrode material is neglected. It should be stated, however, that even though this technique can aid in the discovery of suitable electrolytes for a particular cell chemistry, the true stability will be influenced also by several other properties of the active 
material. Further method improvements which take these parameters into account are certainly welcome.[29]

\section{Acknowledgements}

We acknowledge support from Volkswagen AG and fruitful discussions with Dr. Junli Shi and Dr. Enrica Jochler. We thank Haldor Topsoe for the donation of LNMO material and feedback on the work. We also acknowledge support from the STandUP for Energy consortium.

\section{References}

[1] W. Li, B. Song, A. Manthiram, Chemical Society Reviews, 46 (2017) 3006-3059.

[2] A. Kraytsberg, Y. Ein-Eli, Advanced Energy Materials, 2 (2012) 922-939.

[3] K. Xu, S.P. Ding, T.R. Jow, Journal of The Electrochemical Society, 146 (1999) 4172-4178.

[4] J. Kasnatscheew, M. Evertz, B. Streipert, R. Wagner, R. Klöpsch, B. Vortmann, H. Hahn, S. Nowak, M. Amereller, A.C. Gentschev, P. Lamp, M. Winter, Physical Chemistry Chemical Physics, 18 (2016) 3956-3965.

[5] J. Kasnatscheew, B. Streipert, S. Röser, R. Wagner, I. Cekic Laskovic, M. Winter, Physical Chemistry Chemical Physics, 19 (2017) 16078-16086.

[6] C.F.N. Marchiori, R.P. Carvalho, M. Ebadi, D. Brandell, C.M. Araujo, Chemistry of Materials, 32 (2020) 7237-7246.

[7] P. Peljo, H.H. Girault, Energy \& Environmental Science, 11 (2018) 2306-2309.

[8] P. Ruschhaupt, S. Pohlmann, A. Varzi, S. Passerini, Batteries \& Supercaps, 3 (2020) 698-707.

[9] N. Elgrishi, K.J. Rountree, B.D. McCarthy, E.S. Rountree, T.T. Eisenhart, J.L. Dempsey, Journal of Chemical Education, 95 (2018) 197-206.

[10] P. Dhatarwal, R.J. Sengwa, S. Choudhary, SN Applied Sciences, 1 (2018) 112.

[11] R. Deivanayagam, R. Shahbazian-Yassar, Batteries \& Supercaps, 4 (2021) 596-606.

[12] Y. Qin, H. Lin, Y. Liu, D. Wang, AIP Conference Proceedings, 1890 (2017) 040002.

[13] A.A. Aziz, Y. Tominaga, Polymer Journal, 51 (2019) 61-67.

[14] A. Bhide, J. Hofmann, A. Katharina Dürr, J. Janek, P. Adelhelm, Physical Chemistry Chemical Physics, 16 (2014) 1987-1998.

[15] W. Li, Y. Pang, J. Liu, G. Liu, Y. Wang, Y. Xia, RSC Advances, 7 (2017) 23494-23501.

[16] S. Cho, S. Kim, W. Kim, S. Kim, S. Ahn, Polymers, 10 (2018).

[17] P. Zhu, Y. Zhao, Materials Chemistry and Physics, 233 (2019) 60-67.

[18] T. Ma, G.-L. Xu, Y. Li, L. Wang, X. He, J. Zheng, J. Liu, M.H. Engelhard, P. Zapol, L.A. Curtiss, J. Jorne, K. Amine, Z. Chen, The Journal of Physical Chemistry Letters, 8 (2017) 1072-1077.

[19] P.G. Kitz, M.J. Lacey, P. Novák, E.J. Berg, Analytical Chemistry, 91 (2019) 2296-2303.

[20] K. Xu, M.S. Ding, T. Richard Jow, Electrochimica Acta, 46 (2001) 1823-1827. 
[21] M.P.S. Mousavi, A.J. Dittmer, B.E. Wilson, J. Hu, A. Stein, P. Bühlmann, Journal of The Electrochemical Society, 162 (2015) A2250-A2258.

[22] M.Y. Abeywardana, N. Laszczynski, M. Kuenzel, D. Bresser, S. Passerini, B. Lucht, International Journal of Electrochemistry, 2019 (2019) 8636540.

[23] C. Schultz, S. Vedder, B. Streipert, M. Winter, S. Nowak, RSC Advances, 7 (2017) 27853-27862. [24] N. Mozhzhukhina, E. Flores, R. Lundström, V. Nyström, P.G. Kitz, K. Edström, E.J. Berg, The Journal of Physical Chemistry Letters, 11 (2020) 4119-4123.

[25] J. Alvarado, M.A. Schroeder, M. Zhang, O. Borodin, E. Gobrogge, M. Olguin, M.S. Ding, M. Gobet, S. Greenbaum, Y.S. Meng, K. Xu, Materials Today, 21 (2018) 341-353.

[26] G. Horwitz, E.J. Calvo, L.P. Méndez De Leo, E. de la Llave, Physical Chemistry Chemical Physics, 22 (2020) 16615-16623.

[27] G. Liang, V.K. Peterson, K.W. See, Z. Guo, W.K. Pang, Journal of Materials Chemistry A, 8 (2020) 15373-15398.

[28] B. Aktekin, M.J. Lacey, T. Nordh, R. Younesi, C. Tengstedt, W. Zipprich, D. Brandell, K. Edström, The Journal of Physical Chemistry C, 122 (2018) 11234-11248.

[29] Z. Li, Y. Zhao, W.E. Tenhaeff, Chemistry of Materials, 33 (2021) 1927-1934. 